\title{
Ibuprofen, Carbamazepine and $\beta$-Estradiol Determination Using Thin-Film Microextraction and Gas Chromatography-Mass Spectrometry
}

\author{
Ady Giordano, *,a José Vásquez, ${ }^{b}$ Mauricio Retamal ${ }^{a}$ and Loreto Ascar ${ }^{c}$ \\ ${ }^{a}$ Departamento de Química Inorgánica, Facultad de Química, Pontificia Universidad Católica de \\ Chile, CP22, 7810000 Santiago, Chile \\ ${ }^{b}$ Facultad de Ciencias Naturales, Matemáticas y del Medio Ambiente, Universidad Tecnológica \\ Metropolitana, 7810000 Santiago, Chile \\ ${ }^{c}$ Facultad de Ciencias Químicas y Farmacéuticas, Universidad de Chile, 8380000 Santiago, Chile
}

\begin{abstract}
The use of thin-film microextraction for the extraction of selected pharmaceutical compounds followed by gas chromatography-mass spectrometry detection was evaluated. A segment of polysiloxanes polymer sheet was used as low cost, single use, disposable extraction phase, while Milli-Q water spiked at $20 \mu \mathrm{g} \mathrm{L}^{-1}$ with the analytes was used for the optimization assays. The controlling parameters for the extraction were optimized via experimental design and it was found that an extraction time of $3 \mathrm{~h}$ using a sample volume of $1000 \mathrm{~mL}$ at $\mathrm{pH} 4$ with the addition of $20 \%$ methanol and $20 \%$ sodium chloride provided the greatest extraction efficiency. Recoveries between 67.1 and $85.0 \%$ were achieved, with a repeteability lower than $20 \%$ (expressed as coefficient of variation) and limit of detection ranged from 0.41 and $0.92 \mu \mathrm{g} \mathrm{L}^{-1}$. The proposed method show similar analytical performance when compared to the determination of the analytes using stir bar sorptive extraction.
\end{abstract}

Keywords: thin-film extraction, $\beta$-estradiol, carbamazepine, ibuprofen

\section{Introduction}

A wide variety of pharmaceutical and personal care products (PPCPs) and their metabolites are continuously introduced in the environment through human excretion, washing and manufacturing. ${ }^{1}$ In recent years, the interest in these compounds has increased, with special attention paid to their presence in the aquatic environment. ${ }^{2}$ However, there is little information about the adverse effects of human pharmaceuticals on aquatic organisms. ${ }^{3}$ Indeed it is known that even though the concentrations found in surface water are very low, the constant introduction of low concentrations represents a risk; compounds such as $\beta$-estradiol, carbamazepine and ibuprofen have been shown to have adverse effects on aquatic ecosystems. ${ }^{2}$

A wide range of analytical methods have been developed for the analysis of PPCPs in aqueous samples. High-performance liquid chromatography (HPLC) with ultraviolet (UV) detection methods are commonly used for the determination of PPCPs in several matrices ${ }^{4}$ gas

*e-mail: agiordano@uc.cl chromatography-mass spectrometry (GC-MS) is another good alternative, because it can be used to identify these analytes with low limits of detection (LOD). ${ }^{5}$ However, in aqueous environmental samples the concentrations of PPCPs are very low and extraction and preconcentration steps are required for their determination. Commonly used PPCPs can be easily extracted via solid-phase extraction using a water-wettable, hydrophilic/lipophilic balance, reversed-phase sorbent with proper $\mathrm{pH}$ adjustment, ${ }^{6-9}$ with recoveries up to $107 \% \%^{6}$ on optimum experimental conditions and LOD as low as $1 \mathrm{ng} \mathrm{L}^{-1}$ when coupled with ultra high performance liquid chromatographic system attached to high-resolution mass spectrometry (UHPLC-MS/MS). ${ }^{10}$ Solid-phase microextraction (SPME) ${ }^{11,12}$ and stir bar sorptive extraction (SBSE) ${ }^{13,14}$ have also been proven to be effective for the determination of these analytes in aqueous sample. Recoveries for estrogen compounds range from 50 to $120 \%$ of the spiked concentration when using SPME $^{6}$ and, for SBSE average recoveries of $93.1 \%$ were achieved for selected PPCPs. ${ }^{14}$ Both of these techniques have the advantages of isolate the analytes from potential interfering matrix components and being environmentally friendly; 
since they use little or no solvent during the extraction and analytes can be thermally desorbed. However, SPME commercial fiber is expensive and has a small quantity of polydimethylsiloxane (PDMS) in the coating which limits analyte abdsorption. Commercial SBSE devices afford better recovery values through the use of higher PDMS volumes, but require a special thermal desorption unit for fully solvent-free operation.

Thin-film microextraction (TFME) was introduced by Bruheim et al. ${ }^{15}$ and comprise cheap commercial thin sheets of PDMS cut into different geometries, which increases the surface area-to-volume ratio to improve the recovery of analytes when using PDMS as the extraction phase. ${ }^{16}$ TFME exhibits the same extraction phenomena and extraction selectivities as SPME and SBSE, ${ }^{17}$ but with increased recovery because a higher volume of PDMS comes into contact with the sample. This configuration allows to remain the thickness of the coating or even decreases it, while the volume of the extraction phase increases, which according to the extraction principle will result in lower equilibrium times and higher extraction rates. ${ }^{18}$ TFME has been widely used for the determination of polyaromatic hydrocarbons, ${ }^{19,20}$ pesticides $^{21}$ and endocrine disruptor compounds. ${ }^{22}$

The aim of this study was to evaluate the use of TFME as a cheap and simple extraction technique for the determination of selected PPCPs with acidic, basic and neutral characteristics. An experimental design matrix was used to optimize the variables that determine TFME extraction performance: $\mathrm{pH}$, extraction time, sample volume and the addition of matrix modifiers, such as methanol $(\mathrm{MeOH})$ and sodium chloride $(\mathrm{NaCl})$.

\section{Experimental}

\section{Reagents}

The mixture $\mathrm{N}, \mathrm{O}$-bis(trimethylsilyl)trifluoroacetamide (BSTFA) + chlorotrimethylsilane (TCMS), 99:1 was obtained from Supelco (Bellefonte, PA, USA). Pyridine, carbamazepine, ibuprofen and $\beta$-estradiol (purity ca. 94-99.5\%) were obtained from Sigma-Aldrich (St. Louis, MO, USA). All other solvents and reagents were purchased from Merck (Darmstadt, Germany) in their highest purity. Ultrapure water was obtained using a Direct-Q 3 system from Millipore (Billerica, MA, USA). Nitrogen and helium were purchased from Indura (Santiago, Chile). The PDMS silicone elastomer sheet was obtained from Goodfellow (Oakdale, PA, USA). Stock solutions $\left(1000 \mathrm{mg} \mathrm{L}^{-1}\right)$ for each analyte were prepared monthly and maintained at $4{ }^{\circ} \mathrm{C}$, and all calibration curves and extraction solutions were prepared daily.

\section{GC-MS analysis}

GC-MS was performed on a Clarus 680 GC (PerkinElmer, Waltham, MA, USA) coupled to a Clarus SQ 8T mass detector under electron impact ionization $(70 \mathrm{eV})$ with a 4 min solvent delay and an interface temperature of $230^{\circ} \mathrm{C}$. Samples were separated on an HP-5ms fused-silica capillary column (Agilent Technologies, Santa Clara, CA, USA) with $0.25 \mu \mathrm{m}$ film and $30 \mathrm{~m} \times 0.25 \mathrm{~mm}$ i.d., using helium 6.0 as the carrier gas (flow rate $1.0 \mathrm{~mL} \mathrm{~min}^{-1}$ ). The column temperature was initially held at $100^{\circ} \mathrm{C}$ for $1 \mathrm{~min}$, then programmed to reach $280{ }^{\circ} \mathrm{C}$ at a rate of $20{ }^{\circ} \mathrm{C} \mathrm{min}^{-1}$ with a final hold time of $15 \mathrm{~min}$. The injector temperature was maintained at $280{ }^{\circ} \mathrm{C}$ and the injection volume was $1 \mu \mathrm{L}$ in the splitless mode. Table 1 lists the analyte ions used for the chromatographic analysis; a tolerance of $0.5 \%$ for retention time and $\mathrm{a} \pm 10 \%$ tolerance for relative ion intensities were employed.

Table 1. Main features of the gas chromatography-mass spectrometry (GC-MS) method

\begin{tabular}{lccc}
\hline Analyte & $\begin{array}{c}\text { Retention } \\
\text { time } / \mathrm{min}\end{array}$ & $\begin{array}{c}\text { Target } \\
\text { ion } /(\mathrm{m} / \mathrm{z})\end{array}$ & $\begin{array}{c}\text { Qualifier } \\
\text { ion } /(\mathrm{m} / \mathrm{z})\end{array}$ \\
\hline Ibuprofen & 5.87 & 263 & 117,160 \\
Carbamazepine & 9.14 & 193 & 165,221 \\
及-Estradiol & 10.86 & 285 & 232,416 \\
\hline
\end{tabular}

\section{TFME procedure}

An aliquot of Milli-Q water was spiked with each analyte at a concentration of $20 \mu \mathrm{g} \mathrm{L} \mathrm{L}^{-1}$ and then the $\mathrm{pH}$ of the solution was adjusted to 4 as measured using a WTW pMX 300 pH meter (Weilheim, Germany) by adding HCl. $\mathrm{NaCl}$ and $\mathrm{MeOH}$ were then each added at a concentration of $20 \%$, followed by a $1 \mathrm{~cm}^{2}$ PDMS polymer sheet. The mixture was then stirred using a Heildolph MR 3002 magnetic stirrer (Schwabach, Germany) for $2 \mathrm{~h}$ at $1500 \mathrm{rpm}$. For the desorption step, the polymer film was placed in $\mathrm{MeOH}(2 \mathrm{~mL})$ and stirred for $30 \mathrm{~min}$. The $\mathrm{MeOH}$ was then taken to dryness under nitrogen. Extraction were conducted in duplicate, except for reproducibility and recovery experiments $(n=5)$.

\section{SBSE procedure}

Stir bars coated with PDMS $(0.5 \mathrm{~mm}$ film thickness, $10 \mathrm{~mm}$ length, $24 \mu \mathrm{L}$ PDMS, $0.94 \mathrm{~cm}^{2}$ surface area) were obtained from Gerstel (Mülheim and der Ruhr, Germany) and were used to compare extraction efficiency.

Prior to use, the stir bars were conditioned into a vial containing $20 \mathrm{~mL}$ of methanol. To perform the extraction, the same procedure as the PDMS polymer sheet was 
followed for both extraction and elution of analytes using optimun conditions of TFME method. Between each extraction, the bar was cleaned with additional $20 \mathrm{~mL}$ of methanol for $30 \mathrm{~min}$.

\section{Derivatization procedure}

To derivatize the analytes, the residue was redissolved in pyridine $(25 \mu \mathrm{L})$ and BSTFA + TMCS $(99: 1 ; 50 \mu \mathrm{L})$ were added. The solution was heated at $75^{\circ} \mathrm{C}$ in a sealed mini-vial for $40 \mathrm{~min}$. Ethyl acetate $(100 \mu \mathrm{L})$ was then added after the silylation reaction be completed.

\section{Sample procedure}

Water samples were collected from the Maipo River (Santiago, Chile). The collected water samples were filtered through $0.45 \mu \mathrm{m}$ nylon filters and stored at $4{ }^{\circ} \mathrm{C}$ in dark bottles until analysis.

\section{Results and Discussion}

$\beta$-Estradiol, carbamazepine and ibuprofen were selected as model compound due to their neutral, basic and acidic characteristic, respectively, in order to study the applicability of the method for PPCPs determination.

For the TFME, a $1 \mathrm{~cm}^{2}$ polymer phase was used in Milli-Q water spiked with the analytes at $20 \mu \mathrm{g} \mathrm{L}^{-1}$. Certain variables that affect the extraction performance, such as the temperature and agitation velocity, were not investigated in the present study, with the extraction being performed at ambient temperature, even when increasing the temperature results in a higher extraction efficiency due to an increase in the diffusion coefficient, as has been reported. ${ }^{22}$ With respect to agitation, a speed of $1500 \mathrm{rpm}$ was chosen because more efficient extraction has been observed with greater agitation..$^{23}$ The effect of the sample volume, extraction time and $\mathrm{pH}$ were optimized using a Box-Behnken design with a total of 15 experiments. Table 2 contains the encoded values and their corresponding natural values.

As can be seen in the total response surface in Figure 1, when grouping the responses of all the compounds, the results indicated that increasing the extraction time increased the extraction efficiency, and this result is comparable to

Table 2. Optimization values for the extraction variables

\begin{tabular}{cccccc}
\hline Factor & $\begin{array}{c}\text { Volume / } \\
\mathrm{mL}\end{array}$ & $\mathrm{pH}$ & $\begin{array}{c}\text { Extraction } \\
\text { time / min }\end{array}$ & $\begin{array}{c}\mathrm{NaCl} / \\
\%\end{array}$ & $\begin{array}{c}\mathrm{MeOH} / \\
\%\end{array}$ \\
\hline-1 & 200 & 3 & 60 & 0 & 0 \\
0 & 600 & 4 & 120 & 10 & 10 \\
+1 & 1000 & 5 & 180 & 20 & 20 \\
\hline
\end{tabular}

results obtained when using single variable optimization. ${ }^{22}$ It is possible that the response could increase beyond the selected parameters and an extraction time higher than 180 min could become an optimum condition. In addition, the relationship between the sample volume and extraction time can also be seen in the Pareto chart in Figure 1 as another important factor. This result is reasonable given that extraction is controlled mainly by mass transfer in the solution. When a higher sample volume was used, the preconcentration factor was increased; therefore, the extraction sensitivity was improved. A lower solution $\mathrm{pH}$ was found to enable increased interaction between the acidic analytes and the polymer. However, the PDMS polymer is known to deteriorate at $\mathrm{pH}$ values less than $2 .{ }^{24}$ Table 3 lists the optimum values determined for each variable.

Since the analytes are mid-polar compounds, the use of sample modifiers was considered. The salting out effect was studied because the addition of a salt is known to enhance the interaction of the analytes with the polymer by

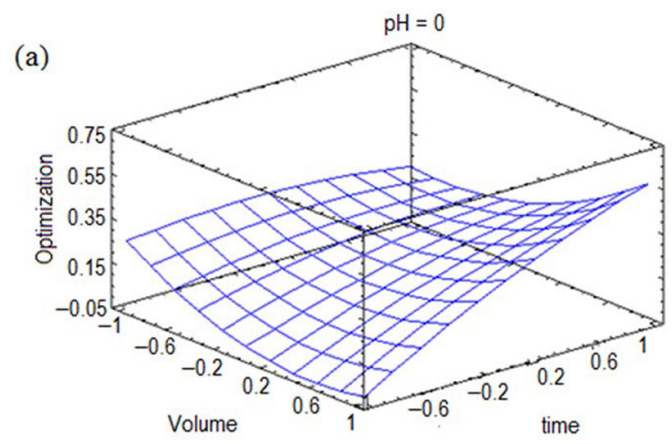

(b)

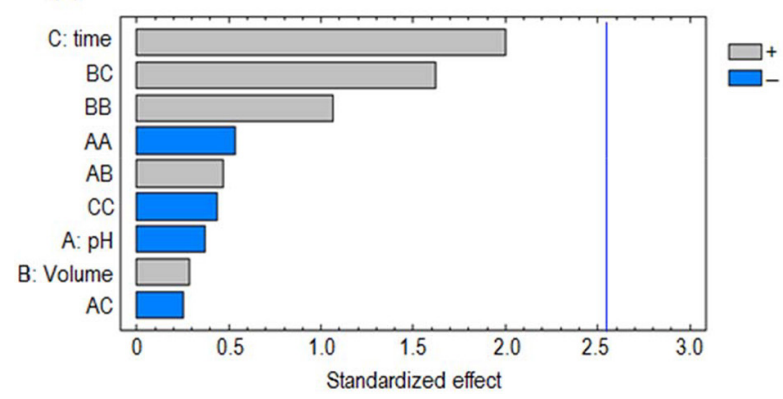

Figure 1. Response surface (a); and Pareto chart (b) for the volume, $\mathrm{pH}$ and extraction time factors.

Table 3. Optimum values for the extraction variables

\begin{tabular}{lcc}
\hline Variable & Encoded value & Natural value \\
\hline Extraction time / min & 1 & 180 \\
Sample volume / $\mathrm{mL}$ & 1 & 1000 \\
$\mathrm{pH}$ & -0.057 & 4 \\
$\mathrm{NaCl} / \%$ & 1 & 20 \\
Methanol / \% & 1 & 20 \\
\hline
\end{tabular}


decreasing their solubilities. ${ }^{21}$ Therefore, $\mathrm{NaCl}$ was added at concentrations up to $20 \%$ (due to salt solubility). The addition of $\mathrm{MeOH}$, a common sample modifier for mid-polar compounds, can increase the solubility of the analytes in the solution. ${ }^{25}$ For optimization of these variables, a $3^{2}$ design was used and the encoded and natural values are also listed in Table 2. It can be seen in the Pareto chart (Figure 2) that both of the modifiers were significant variables. The optimum values are listed in Table 3 and show that increasing both the $\mathrm{NaCl}$ and $\mathrm{MeOH}$ concentrations increased the extraction response; however, the response surface (Figure 2) show just a slightly increment when adding $20 \% \mathrm{MeOH}$.

For all of these experiments, a well-known desorption step was employed. $\mathrm{MeOH}$ was chosen because it does not damage the polymer phase or cause swelling of the PDMS. ${ }^{23}$ Desorption time was optimized between 10 to 30 min of agitation at high speed and 30 min was chosen during the optimization of the desorption time agitation. Under this condition, no carryover effect was observed.

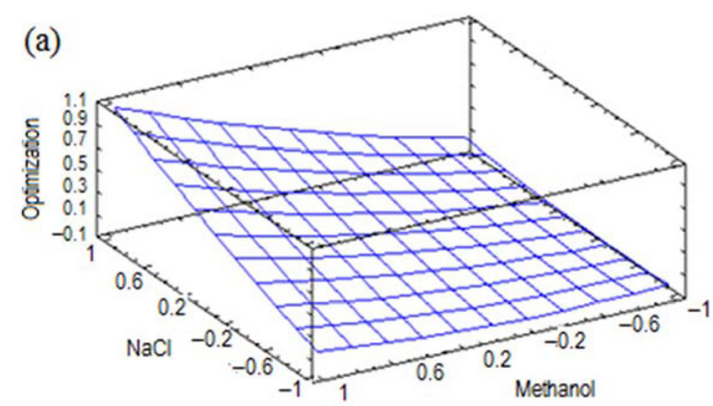

(b)

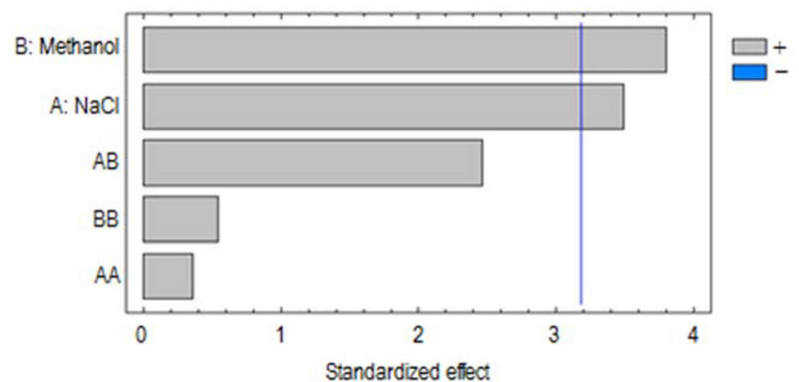

Figure 2. Response surface (a); and Pareto chart (b) for percentages of $\mathrm{NaCl}$ and methanol factors.

The quantity of analyte that can be extracted using thinfilm extraction is determined by the volume of the extraction phase. ${ }^{15}$ Therefore, increasing the size of the polymer film used for the extraction was also evaluated. A $2 \mathrm{~cm}^{2}$ polymer sheet was introduced to the analyte solution and the same two experimental designs were performed. Similar trends to those observed using the $1 \mathrm{~cm}^{2}$ polymer phase were observed with the larger polymer sheet and the optimum conditions were again those presented in Table 2. Notably, there was an increase in the responses for carbamazepine and $\beta$-estradiol, reflecting the fact that analyte recovery is also dependent on the analyte/polymer phase ratio and the octanol-water partition coefficient $\left(\mathrm{K}_{\mathrm{OW}}\right)$ of the analyte..$^{15}$ However, no effect on the recovery of ibuprofen when using the larger polymer phase was observed, meaning that this analyte extraction was already exhaustive when using less volume of PDMS.

\section{Analytical features}

For quantitative analysis, it is not necessary that the analytes are completely extracted, as long as the extraction is carefully timed and the experimental conditions remain constant. ${ }^{26}$ Calibration curves were constructed usign seven points for analytes concentrations ranging from 1 to $50 \mu \mathrm{g} \mathrm{L}^{-1}$ in aqueous solutions $(n=2)$. The resulting calibration equations are presented in Table 4, along with their associated limits of detection and quantification (values were calculated using the parameters of the calibration curves).

Water samples fortified at $20 \mu \mathrm{g} \mathrm{L}-1$ for all analytes were used to evaluate accuracy. 5 samples were prepared and extracted the same day for recovery tests and repeatability assays. Both results are shown in Table 4. Intermediate precision was evaluated as the coefficient of variance of the analysis of water samples on three differents days using five repetitions and the values obtained were between 8.4 and $20.0 \%$. Note that the PDMS phase was not reused and the polymer sheet was not homogenous. Despite these issues, the obtained values are similar to those reported by other authors using similar extraction processes. ${ }^{25}$

However, when comparing the proposed methodology with other reported in the literature, lower LOD are described. Neng et al. ${ }^{5}$ use polyethylene sorptive bars with large volume injection GC-MS, achieving lower LOD with no derivatization step. On the other hand, when using UPLC-MS/MS for quantification, LOD of $1 \mathrm{ng} \mathrm{L}^{-1}$ are reported. However, in Celano et al..$^{9}$ work is used a combination of extraction methodologies. To be able to achieve such low LOD, the developed TFME methodology needs to be coupled with a more sensitive detection method. However, the simplicity of the extraction, the cheap extraction phase and good analytical performance make the proposed methodology a suitable alternative for determinations of the selected PPCPs.

\section{Comparison with SBSE methodology}

A comparison of the extraction efficiency was made between TFME and SBSE using five extractions of water 
Table 4. Analytical parameters for the analytes in aqueous samples

\begin{tabular}{|c|c|c|c|c|c|c|}
\hline \multirow[b]{2}{*}{ Analyte } & \multirow[b]{2}{*}{ Calibration curve } & \multirow[b]{2}{*}{$\begin{array}{l}\text { Correlation } \\
\text { coefficient }\end{array}$} & \multirow[b]{2}{*}{$\begin{array}{c}\mathrm{LOD} / \\
\left(\mu \mathrm{g} \mathrm{L}^{-1}\right)\end{array}$} & \multirow[b]{2}{*}{$\begin{array}{l}\mathrm{LOQ} / \\
\left(\mu \mathrm{g} \mathrm{L}^{-1}\right)\end{array}$} & \multicolumn{2}{|c|}{ Accuracy and repeatability } \\
\hline & & & & & $\begin{array}{l}\text { Coefficient of } \\
\text { variation / \% }\end{array}$ & Recovery / \% \\
\hline$\beta$-Estradiol & $\mathrm{y}=0.86 \mathrm{E} 4 \mathrm{x}+5.133$ & 0.9852 & 0.41 & 1.36 & 11.8 & 85.0 \\
\hline Carbamazepine & $\mathrm{y}=4.21 \mathrm{E} 4 \mathrm{x}+1.13 \mathrm{E} 3$ & 0.9809 & 0.72 & 2.40 & 12.5 & 67.1 \\
\hline Ibuprofen & $\mathrm{y}=2.72 \mathrm{E} 5 \mathrm{x}+9.31 \mathrm{E} 4$ & 0.9914 & 0.92 & 3.07 & 6.4 & 79.5 \\
\hline
\end{tabular}

${ }^{\mathrm{a}}$ Concentration level $20 \mu \mathrm{g} \mathrm{L}{ }^{-1}$ and $\mathrm{n}=5$. LOD: Limit of detection; LOQ: limit of quantification.

samples spiked at $20 \mu \mathrm{g} \mathrm{\textrm {L } ^ { - 1 }}$ with optimized conditions. The results are presented in Figure 3. Under similar extraction conditions, similar recoveries are obtained when using SBSE instead of a polymer sheet; however, for carbamazepine, a slightly increase in efficiency can be observed probably due to the polymer composition not being $100 \%$ PDMS as established by the manufacturer, which allows a better interaction between the analytes and the extraction phase. In addition to the recovery values, it was observed similarities between the repeatability of both methods.

\section{Real samples}

The proposed method was applied to the analysis of real aqueous environmental samples obtained from 5 sites in Maipo River. All samples showed results under LOD. To verify the accuracy of the method, recovery experiments

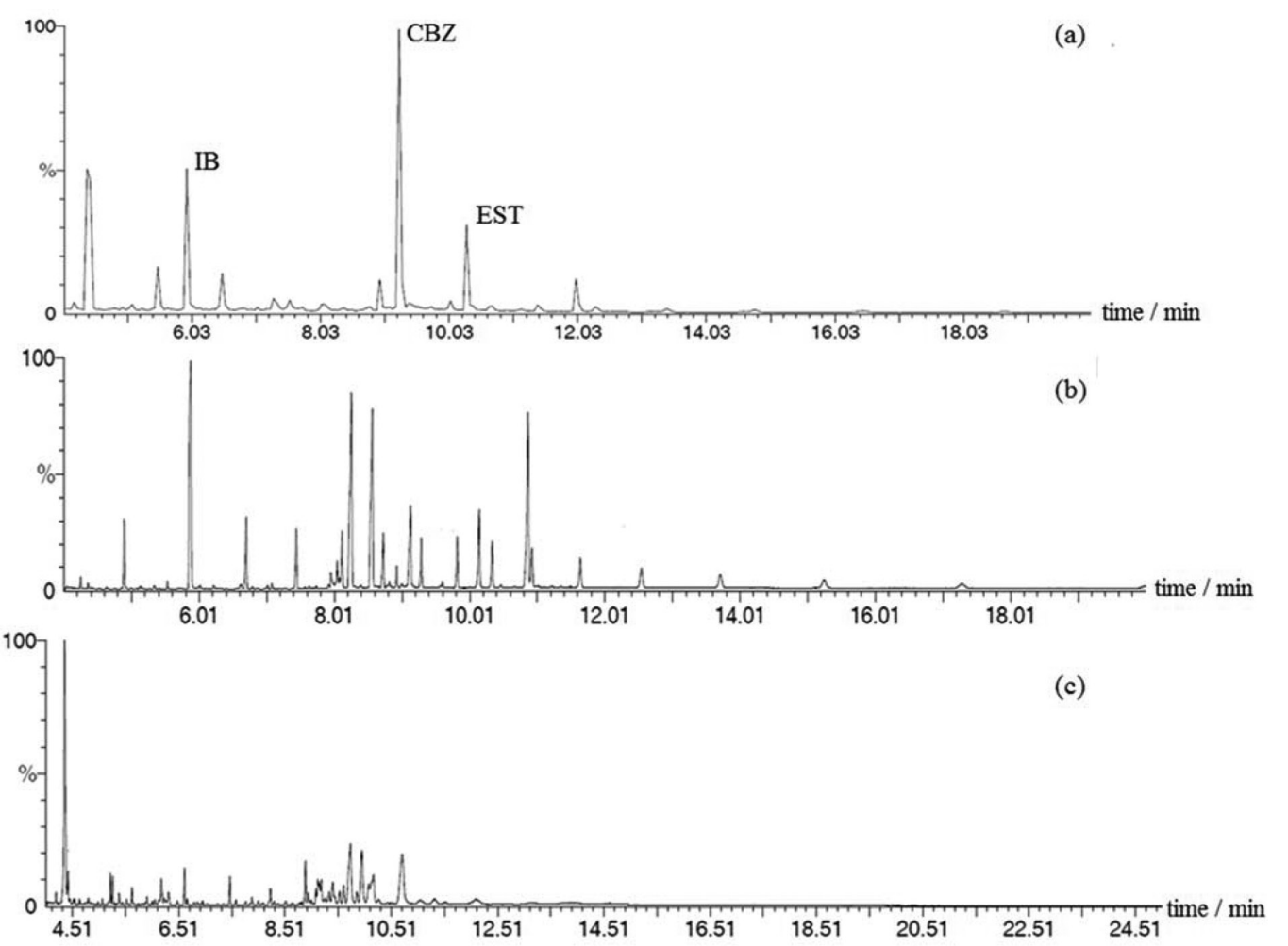

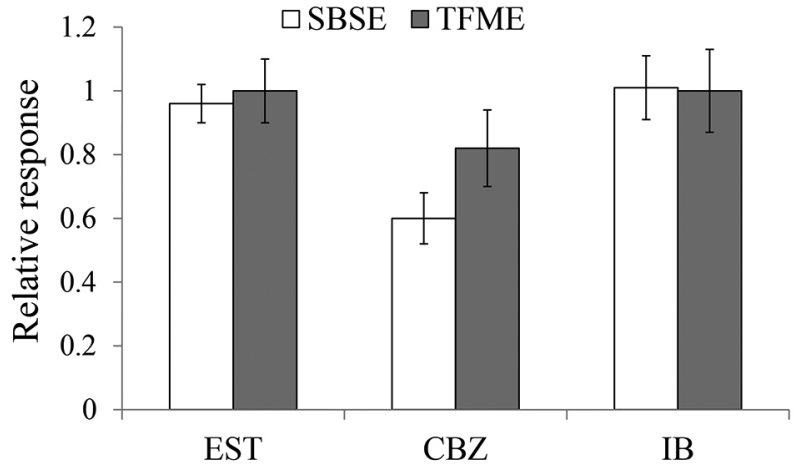

Figure 3. Relative response of analytes when determined with stir bar sorptive extraction (SBSE) and the proposed methodology. CBZ: Carbamazepine; EST: $\beta$-estradiol; IB: ibuprofen; TFME: thin-film microextraction.

were carried out by the analysis of spiked samples, with recoveries between $62.7-81.0 \%$ (Figure 4). The signal identification was made by comparison of standards in pure (a) 
solvent, while the recovery of the analytes was calculated using calibration curves in sample matrix.

\section{Conclusions}

The extraction/preconcentration of selected PPCPs using TFME was demonstrated. The use of experimental design reduced the experimental work and gave reliable information regarding the parameters controlling the extraction performance. We found that the sample volume, extraction time and the quantity of added $\mathrm{MeOH}$ and $\mathrm{NaCl}$ were of relevance to the extraction efficiency and should be particularly controlled. The use of TFME for PPCP analysis has the advantages of being cheap, easy, environmental friendly and a suitable alternative approach to determine pharmaceutical compounds in water samples.

\section{Acknowledgments}

The authors thank Project Fondecyt No.11121237 for their financial support.

\section{References}

1. Ellis, J. B.; Environ. Pollut. (Oxford, U. K.) 2001, 144, 184.

2. Buchberger, W. W.; J. Chromatogr. A 2011, 1218, 603.

3. Richardson, B.; Lam, P. K. S.; Martin, M.; Mar. Pollut. Bull. 2005, 509, 13.

4. Ahn, Y. G.; Shin, J. H.; Kim, H. Y.; Khim, J.; Lee, M. K.; Hong, J.; Anal. Chim. Acta 2007, 603, 67.

5. Neng, N. R.; Nogueira, M. F.; Anal. Bioanal. Chem. 2012, 402, 1355.

6. Ferrer, I.; Zweigenbaum, J. A.; Thurman, E. M.; J. Chromatogr. A 2010, 1217, 5674.

7. Batt, A. L.; Kostich, M. S.; Lazorchak, J. I. M.; Anal. Chem. 2008, 80, 5021.

8. Kasprzyk-Hordern, B.; Dinsdale, R. M.; Guwy, A. J.; Anal. Bioanal. Chem. 2008, 391, 1293.
9. Celano, R.; Piccinelli, A. L.; Campone, L.; Rastrelli, L.; J. Chromatogr. A 2014, 1355, 26.

10. Rodil, R.; Quintana, J. B.; Lopez-Mahia, P.; MuniateguiLorenzo, S.; Prada-Rodriguez, D.; J. Chromatogr. A 2009, 1216, 2958.

11. Peñalver, A.; Pocurull, E.; Borrull, F.; Marcé, R. M.; J. Chromatogr. A 2002, 964, 153.

12. Carpinteiro, J.; Quintana, J. B.; Rodriguez, I.; Carro, A. M.; Lorenzo, R. A.; Cela, R.; J. Chromatogr. A 2004, 1056, 179.

13. Kawaguchi, M.; Ito, R.; Sakui, N.; Okanouchi, N.; Saito, K.; Nakazawa, H.; J. Chromatogr. A 2006, 1105, 140.

14. Tan, B. L. L.; Hawker, D. W.; Müller, J. F.; Tremblay, L. A.; Chapman, H. F.; Water Res. 2008, 42, 404.

15. Bruheim, I.; Liu, X.; Pawliszyn, J.; Anal. Chem. 2003, 75, 1002.

16. Jiang, R.; Pawliszyn, J.; TrAC, Trends Anal. Chem. 2012, 39, 245.

17. Pawliszyn, J.; J. Chromatogr. Sci. 2000, 38, 270.

18. Qin, Z.; Mok, S.; Ouyang, G.; Dixon, D. G.; Pawliszyn, J.; Anal. Chim. Acta 2010, 667, 71.

19. Qin, Z.; Bragg, L.; Ouyang, G.; Pawliszyn, J.; J. Chromatogr. A 2008, 1196, 89.

20. Wei, F.; Zhang, F.; Liao, H.; Dong, X.; Li, Y.; Chen, H.; J. Sep. Sci. 2011, 34, 331.

21. Cai, P. S.; Li, D.; Chen, J.; Xiong, C. M.; Ruan, J. L.; Food Chem. 2015, 173, 1158.

22. Giordano, A.; Richter, P.; Ahumada, I.; Talanta 2011, 85, 2425.

23. Richter, P.; Leiva, C.; Choque, C.; Giordano, A.; Sepúlveda, B.; J. Chromatogr. A 2009, 1216, 8598.

24. Prieto, A.; Basauri, O.; Usobiaga, R.; Fernandez, L. A.; Etxebarria, N.; Zuloaga, O.; J. Chromatogr. A 2010, 1217, 2642.

25. Liu, W.; Hu, Y.; Zhao, J.; Xu, J.; Guan, Y.; J. Chromatogr. A 2005, 1095, 1.

26. Lambropoulou, D. A.; Giokas, D. L.; Sakkas, V. A.; Albanis, T. A.; Karayannis, M. I.; J. Chromatogr. A 2002, 967, 243.

Submitted: October 30, 2015

Published online: February 18, 2016 